\title{
The Octahedral Manipulator Revisited
}

\author{
Nicolás Rojas, Júlia Borràs, and Federico Thomas
}

\begin{abstract}
In most practical implementations of the GoughStewart platform, the octahedral form is either taken as it stands or is approximated. The kinematics of this particular instance of the Gough-Stewart platform, commonly known as the octahedral manipulator, has been thoughtfully studied. It is well-known, for example, that its forward kinematics can be solved by computing the roots of an octic polynomial and its singularities have a simple geometric interpretation in terms of the intersection of four planes in a single point. In this paper, using a distance-based formulation, it is shown how these properties can be derived without relying neither on variable eliminations nor trigonometric substitutions. Moreover, thanks to this formulation, a family of platforms kinematically equivalent to the octahedral manipulator is obtained. Herein, two Gough-Stewart parallel platforms are said to be kinematically equivalent if there is a one-to-one correspondence between their squared leg lengths for the same configuration of their moving platforms with respect to their bases. If this condition is satisfied, it can be shown that both platforms have the same assembly modes and their singularities, in the configuration space of the moving platform, are located in the same place.
\end{abstract}

\section{INTRODUCTION}

The Stewart-Gough platform consists of a fixed base and a moving platform connected by six ball-ended extensible legs [2]. While the kinematics analysis of the general case, that is, that in which the ball-and-socket joints are arbitrarily located on the base and the platform, is very complex, it gets greatly simplified when some of these joints, either on the base or the platform, coalesce and/or are made to be collinear or coplanar. In other words, placing constraints on the geometrical structure of the general Stewart-Gough platform offers the opportunity for obtaining a simple formulation for its forward kinematics and a simple geometrical interpretation for its singularities. The maximum simplification is obtained when all the ball-and-socket joints coalesce into only three multiple spherical joints both in the base and the platform. Only three possibilities arise whose topologies are represented in Fig. 1. These three platforms are known as the three 3-3 StewartGough platforms for obvious reasons.

One of the 3-3 Stewart-Gough platforms consists of six double-ball-ended legs thereby forming a zigzag pattern. For symmetry reasons, this topology is either taken as it stands or is approximated in most implementations of the StewartGough platform. Since the 12 lines that join the double-balljoints can be interpreted as the eight triangular faces of an octahedron, the term octahedral manipulator was coined in [3] to name it.

The authors are with the Institut de Robòtica i Informàtica Industrial (CSIC-UPC), Llorens Artigas, 4-6, 08028, Barcelona, Spain \{nrojas, jborras, fthomas\}@iri.upc.edu
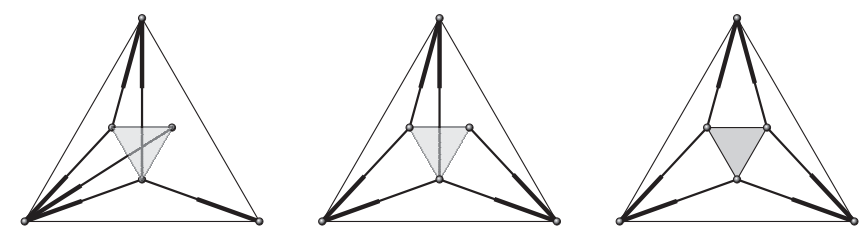

Fig. 1. The three possible topologies for a 3-3 Stewart-Gough platform. The lower one corresponds to the octahedral manipulator.

Clearly, it is advantageous to have multiple spherical joints sharing the same center of rotation in a parallel manipulator to simplify its kinematics. However, difficulties always arise in constructing such spherical joints. There have been several attempts to construct them (see [4] and the references therein), but none of them use off-the-self mechanical elements. Another disadvantage of this kind of joints is that the range of action of the leg actuators is reduced because of the risk of mechanical interference. In [5], kinematic substitutions are introduced to provide a way around this problem where is it shown, for example, that the manipulator appearing in Fig. 2(a), that avoids the doubleball-joints in the base, is kinematically equivalent to the octahedral manipulator. This particular arrangement of joints is also known as the triple arm mechanism [6].

Most implementations avoid the difficulty of constructing multiple spherical joints by approximating them with a collection of single spherical joints with small offsets between them, as shown in Fig. 2(b). Such offsets change the kinematics of the mechanism, resulting in one of two possible problems, as pointed out in [4]. First, if the offsets are included in the kinematics of the mechanism, the kinematic equations may become very complex and thus very difficult to solve. Second, if the offsets are neglected, thus simplifying the kinematic equations, errors arise. These errors may have a significant impact in precision applications, or in manipulators such as the Tetrobot [7] that consists in stacking multiple octahedral manipulators resulting in the accumulation of errors if such offsets are introduced and neglected.

The modification of the octahedral manipulator proposed by Stoughton and Arai consist in separating the six doubleball joints alternatively inward and outward radially [8], as shown in Fig. 2(c). Each double-ball-joint is separated by the same amount into a pair of spherical joints whose centers are equidistant to the original center. In this paper, we show that, if this six double-ball joints are alternatively separated not radially but following the edges of the base and platform triangles, as shown in Fig. 2(d), the resulting manipulator 


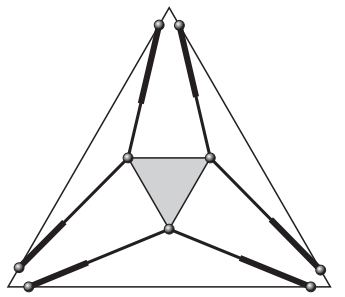

(a)

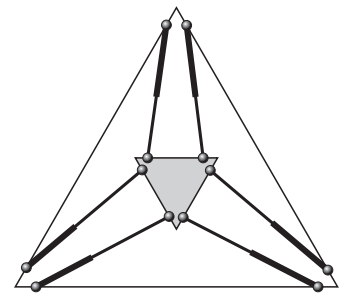

(b)

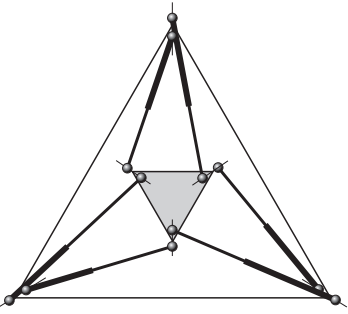

(c)

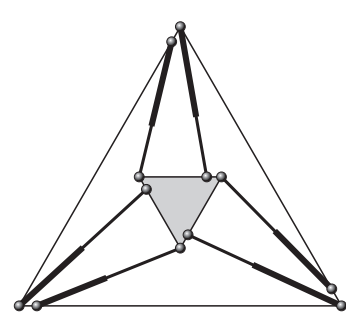

(d)

Fig. 2. The triple arm mechanism (a), the standard approximation to the octahedral manipulator that avoids all double-ball-joints (b), the Stoughton-Arai approximation intended to also improve the dexterity of the manipulator (c), and the Griffis-Duffy modification (d).

is kinematically equivalent to the original octahedral one. This fact was already acknowledged by Griffis and Duffy in [9] (without giving an explicit formulation) but it has been overlooked in subsequent publications where alternatives to avoid these joints are discussed [5]. The formal prove to this fact can be easily derived through a formulation of the kinematics of the octahedral manipulator fully expressed in terms of distances.

This paper is organized as follows. Section II presents a novel closure condition for octahedra, that is, a condition that is satisfied if, and only if, an octahedron can be assembled with the desired edge lengths. Section III briefly reviews the proposed approaches to solve the forward kinematic of the octahedral manipulator and shows how its characteristic octic polynomial corresponds to the closure condition derived in the previous section. Then, using this formulation, it is shown that, when there is an affine relationship between the squared leg lengths of two platforms, a one-to-one-correspondence exits between the coefficients of their characteristic polynomials or, equivalently, between the solutions to their forward kinematics. Section IV deals with the singularities of the octahedral manipulator and the relationship between the singularity locus of two platforms whose squared leg lengths are affine linearly related. In Section V, the geometric transformations that lead to affine relationship between the squared of the leg lengths is derived. A family of parallel platforms kinematically equivalent to the octahedral manipulator is thus obtained. One of its members has no doubleball-joints. Section VI analyzes this case through an example. Finally, Section VII summarizes the main results.

\section{DISTANCE-BASED CLOSURE CONDITION FOR AN OCTAHEDRON}

Let us consider the octahedron in Fig. 3(left). It can be decomposed into two bananas (two sets of two tetrahedra sharing one face). Then, let us consider the banana in Fig. 3(right-top). For this banana, it can be proved that

$$
D(5,4,1,2 ; 5,4,1,6)= \pm \sqrt{D(5,4,1,2) D(5,4,1,6)}
$$

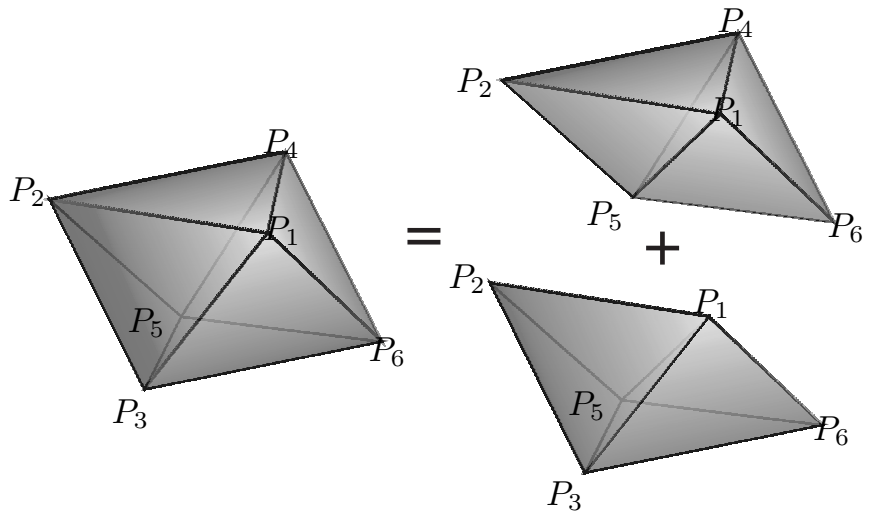

Fig. 3. An octahedron can be decomposed into two bananas. In these two bananas, the squared edge lengths $s_{1,6}$ and $s_{3,4}$ are unknown, but for each banana $s_{1,6}$ and be expressed as a function of $s_{3,4}$. Equating both solutions for $s_{3,4}$ a closure condition for the original octahedron in terms of $s_{1,6}$ is thus obtained.

where

$$
D\left(i_{1}, \ldots, i_{n} ; j_{1}, \ldots, j n\right)=\left|\begin{array}{cccc}
0 & 1 & \ldots & 1 \\
1 & s_{i_{1}, j_{1}} & \cdots & s_{i_{1}, j_{n}} \\
\vdots & \vdots & \ddots & \vdots \\
1 & s_{i_{n}, j_{1}} & \ldots & s_{i_{n}, j_{n}}
\end{array}\right|,
$$

and

$$
D\left(i_{1}, \ldots, i_{n}\right)=D\left(i_{1}, \ldots, i_{n} ; i_{1}, \ldots, i_{n}\right)
$$

are Cayley-Menger determinants, $s_{i, j}$ being the squared distance between $P_{i}$ and $P_{j}$ (see [1] for details on the derivation of this formula).

Applying also this result to the banana in Fig. 3(rightbottom), we get

$$
D(3,5,1,2 ; 3,5,1,6)= \pm \sqrt{D(3,5,1,2) D(3,5,1,6)}
$$

Equations (1) and (2) depend on both $s_{2,6}$ and $s_{1,5}$, but this dependency is linear for $s_{2,6}$. Then, by solving them for $s_{2,6}$ and equating the results, a scalar equation in a single variable, $s_{1,5}$, is obtained:

$$
\begin{array}{r}
\frac{1}{-16 A_{3,5,1} A_{5,1,4}}\left[A_{5,1,4} \Psi_{3,5,1,2,6}-A_{3,5,1} \Psi_{5,1,4,2,6}\right. \\
+288( \\
+A_{5,1,4} V_{3,5,1,2} V_{3,5,1,6} \\
\left.\left.\mp A_{3,5,1} V_{5,1,4,2} V_{5,1,4,6}\right)\right]=0 .
\end{array}
$$


where $A_{i, j, k}=\frac{1}{4} D(i, j, k)$ is the squared area of the triangle $P_{i} P_{j} P_{k}, V_{i, j, k, l}=\frac{1}{6} \sqrt{D(i, j, k, l)}$ is the volume of the tetrahedron $P_{i} P_{j} P_{k} P_{l}$, and $\Psi_{i, j, k, l, m}$ is a polynomial function in $s_{i, j}, s_{i, k}, s_{i, l}, s_{i, m}, s_{j, k}, s_{j, l}, s_{j, m}, s_{k, l}$, and $s_{k, m}$

Equation (3) is satisfied if, and only if, the considered octahedron can be assembled with its assigned edge lengths. Actually, the different real roots of this equation for $s_{1,5}$ correspond to the different ways in which the octahedron can be assembled. Note that two other equivalent conditions could be derived for $s_{2,6}$ and $s_{3,4}$ by decomposing the octahedron into different sets of bananas.

\section{FORWARD KINEMATICS OF THE OCTAHEDRAL MANIPULATOR}

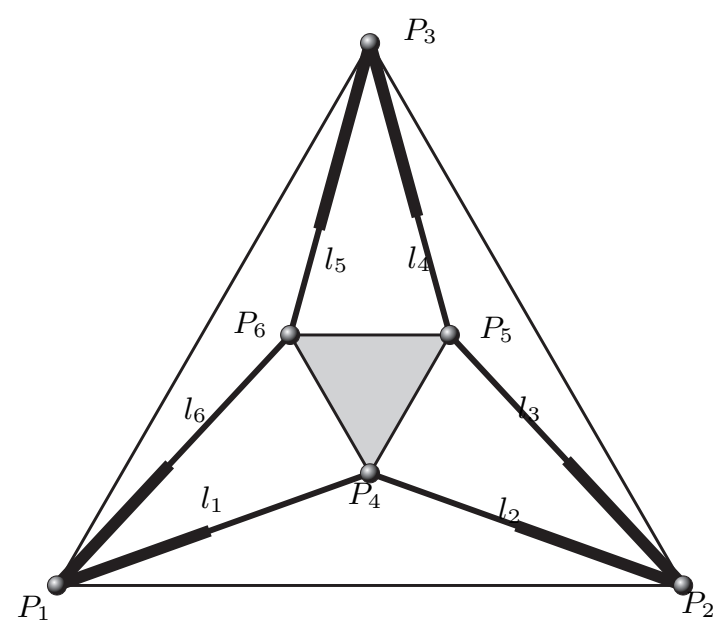

Fig. 4. Octahedral manipulator and associated notation. The triangles $P_{1} P_{2} P_{3}$ and $P_{4} P_{5} P_{6}$ are the base and platform, respectively

The forward kinematics problem is to find all poses of the platform (relative to the base) that are compatible with the six specified leg lengths. During the late 80's and early 90 's several researchers successfully addressed it giving numerical procedures that involve finding the roots of an eighth-degree univariate polynomial. In [11], Nanua et al. derived such a polynomial through resultant elimination and tangent-half-angle substitution techniques. A similar result, based on three spherical four-bar linkages, was obtained by Griffis and Duffy in [12]. An alternative method was also developed by Innocenti and Parenti-Castelli in [13]. In all cases the polynomial variable is the tangent of onehalf the angle defined by the plane supporting $P_{1} P_{2} P_{4}$ (alternatively $P_{2} P_{3} P_{5}$, or $P_{3} P_{1} P_{6}$ ) and the base plane. More recently, Akçali and Mutlu revisited the problem —also using resultant elimination and tangent-half-angle substitution techniques - with the aim of reducing the computational cost of evaluating the resulting univariate polynomial [14]. Finally, it is worth to mention that the forward kinematics of the octahedral manipulator has also been solved locally using Newton-Raphson iterative schemes. Liu et al. [15], Ku [16], and Song and Kwon [17] propose different formulations to this end.
Using the closure condition for an octahedron, derived in the previous section, and trilaterion [10], a simple procedure for solving the forward kinematics problem is obtained. Indeed, consider the octahedral manipulator in Fig. 4 (the triangles $P_{1} P_{2} P_{3}$ and $P_{4} P_{5} P_{6}$ are the base and platform, respectively). This robot can be assembled if, and only if, equation (3) is satisfied for real values of $s_{1,5}$. Thus, the roots of this equation determine the assembly modes of the considered manipulator. These roots can be obtained by computing the roots of the 8th order polynomial that results after twice squaring it. For each of these real roots, we can determine the spatial position of the three points of the platform by computing, for example, the following sequence of trilaterations: computing $\mathbf{p}_{1,5}$ from $\mathbf{p}_{1,2}$ and $\mathbf{p}_{1,3}$, then $\mathbf{p}_{1,4}$ from $\mathbf{p}_{1,2}$ and $\mathbf{p}_{1,5}$, and finally $\mathbf{p}_{1,6}$ from $\mathbf{p}_{1,4}$ and $\mathbf{p}_{1,5}$. This leads to up to eight locations for $P_{6}$. Those locations that satisfy the distance imposed by the leg connecting $P_{3}$ and $P_{6}$ correspond to valid assembly modes.

Now, let us suppose that, for a generic configuration of the moving platform with respect to the base, the location of the joints are modified so that the lengths of the legs for the new locations, say $m_{1}, m_{2}, \ldots, m_{6}$, are related to those of the original ones, $l_{1}, l_{2}, \ldots, l_{6}$, through the relation:

$$
\left(\begin{array}{c}
m_{1}^{2} \\
m_{2}^{2} \\
\vdots \\
m_{6}^{2}
\end{array}\right)=\mathbf{A}\left(\begin{array}{c}
l_{1}^{2} \\
l_{2}^{2} \\
\vdots \\
l_{6}^{2}
\end{array}\right)+\mathbf{b}
$$

where $\mathbf{A}$ and $\mathbf{b}$ are a constant matrix and a constant vector, respectively. Then, if such a modification on the location of the joints exists, the resulting platform will have the same forward kinematics as the original one in the sense that there will be a one-to-one correspondence between the coefficients of their associated octic polynomials through (4). The effect of this kind of joint location modifications on the singularities of the moving platform is discussed in the next section.

\section{Singularities}

For a general Stewart-Gough platform, the linear actuators' velocities, $\left(\dot{l}_{1}, \dot{l}_{2}, \ldots, \dot{l}_{6}\right)$, can be expressed in terms of the platform velocity vector $(\mathbf{v}, \boldsymbol{\Omega})$ as follows:

$$
\operatorname{diag}\left(l_{1}, \ldots, l_{6}\right)\left(\begin{array}{c}
\dot{l}_{1} \\
i_{2} \\
\vdots \\
i_{6}
\end{array}\right)=\mathbf{J}\left(\begin{array}{c}
\mathbf{v} \\
\boldsymbol{\Omega}
\end{array}\right)
$$

where $\mathbf{J}$ is the matrix of normalized Plücker coordinates of the six leg lines. The parallel singularities of the platform are those configurations in which $\operatorname{det}(\mathbf{J})=0$. This algebraic condition have a simple geometric interpretation for the octahedral manipulator. Indeed, according to Fig. 4, when the supporting planes of the triangles $P_{1} P_{2} P_{4}, P_{2} P_{3} P_{5}, P_{3} P_{1} P_{6}$, and $P_{4} P_{5} P_{6}$ intersect in a single point, the manipulator is in a singular pose [19]. Alternatively, this condition can be 
expressed as [21]:

$$
\begin{aligned}
& D(1,2,4,5) D(3,4,5,6) D(1,2,3,6) \\
& \quad=D(1,2,3,4) D(2,4,5,6) D(1,3,5,6)
\end{aligned}
$$

Now, as in the previous section, let us suppose that the location of the joints are modified so that the lengths of the legs in their new locations are related to those of the original legs through the relation (4). Differentiating (4) with respect to time and substituting (5) in the result, we get

$$
\operatorname{diag}\left(d_{1}, \ldots, d_{6}\right)\left(\begin{array}{c}
\dot{d}_{1} \\
\dot{d}_{2} \\
\vdots \\
\dot{d}_{6}
\end{array}\right)=\mathbf{A J}\left(\begin{array}{c}
\mathbf{v} \\
\boldsymbol{\Omega}
\end{array}\right)
$$

Then, if a modification in the location of the joints satisfying (4) exists, the singularities of the resulting platform are those configurations in which $\operatorname{det}(\mathbf{A J})=\operatorname{det}(\mathbf{A}) \operatorname{det}(\mathbf{J})=0$. In other words, the resulting platform will have the same singularities as the original one provided that $\operatorname{det}(\mathbf{A}) \neq 0$. As a consequence, a modification in the location of the joints satisfying (4) leaves the singularities of the moving platform unaltered. Next section presents the geometric transformations that satisfy the algebraic condition (4).

\section{DERIVING KINEMATICALly EQUIVALENT MANIPULATORS}

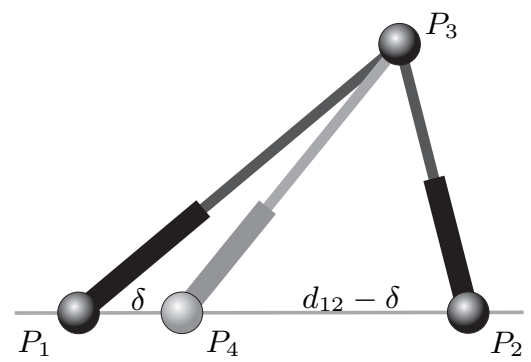

Fig. 5. The squared distance $s_{3,4}$ depends affine linearly on $s_{1,3}$ and $s_{2,3}$ provided that $P_{4}$ lies in the line defined by $P_{1} P_{2}$.

Let us take two legs in an octahedral manipulator sharing a double-ball-joint and let us introduce an offset in the location of one of the other end spherical joints, as shown in Fig. 5. Since the Cayley-Menger determinant of $P_{1}, P_{2}, P_{3}$, and $P_{4}$ vanishes because they are coplanar, $D(1,2,3,4)=0$ or, equivalently,

$$
\delta s_{2,3}+\left(d_{1,2}-\delta\right) s_{1,3}-d_{12} s_{3,4}-d_{1,2} \delta\left(d_{1,2}-\delta\right)=0 .
$$

Note that $s_{3,4}$ depends affine linearly on $s_{1,3}$ and $s_{2,3}$. Then, if the spherical joint centered at $P_{1}$ is moved to $P_{4}$, the resulting leg lengths, for any configuration of the moving platform, can be expressed in terms of the original leg lengths as in (4). Thus, it can be said that the introduced offset does not change the kinematics of the original octahedral manipulator.

It is possible to repeat the above operation on the remaining couples of legs sharing a double-ball-joint. A family of
Stewart platforms kinematically equivalent to the octahedral manipulator is thus obtained. Unfortunately, all members of this family include at least one double-ball-joint. Nevertheless, it is interesting to realize that these offsets can also be introduced simultaneously, not only sequentially. The details of how this operation is performed can be found in [20]. Then, if an offset is simultaneously introduced for the six sets of two legs sharing a double-ball-joint, all joints are split into single spherical joints. The result is the 6-6 platform appearing in Fig. 6.

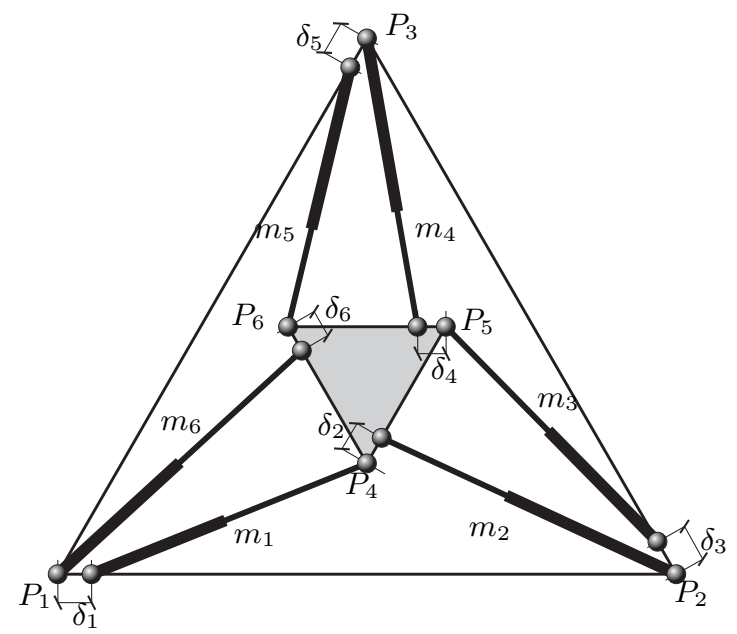

Fig. 6. Contrarily to what happens to the Stoughton-Arai approximation, the proposed modification lead to a 6-6 platform kinematically equivalent to the octahedral manipulator.

According to Fig. 6 and the results in [20], the affine relation between leg lengths of the resulting 6-6 platform and the original octahedral manipulator can be expressed as:

$$
\left(\begin{array}{c}
m_{1}^{2} \\
m_{2}^{2} \\
m_{3}^{2} \\
m_{4}^{2} \\
m_{5}^{2} \\
m_{6}^{2}
\end{array}\right)=\mathbf{A}\left(\begin{array}{l}
l_{1}^{2} \\
l_{2}^{2} \\
l_{3}^{2} \\
l_{4}^{2} \\
l_{5}^{2} \\
l_{6}^{2}
\end{array}\right)-\mathbf{b}
$$

where

$\mathbf{A}=\left(\begin{array}{cccccc}\frac{d_{12}-\delta_{1}}{d_{12}} & \frac{\delta_{1}}{d_{12}} & 0 & 0 & 0 & 0 \\ 0 & \frac{d_{45}-\delta_{2}}{d_{45}} & \frac{\delta_{2}}{d_{45}} & 0 & 0 & 0 \\ 0 & 0 & \frac{d_{23}-\delta_{3}}{d_{23}} & \frac{\delta_{3}}{d_{23}} & 0 & 0 \\ 0 & 0 & 0 & \frac{d_{56}-\delta_{4}}{d_{56}} & \frac{\delta_{4}}{d_{56}} & 0 \\ 0 & 0 & 0 & 0 & \frac{d_{13}-\delta_{5}}{d_{13}} & \frac{\delta_{5}}{d_{13}} \\ \frac{\delta_{6}}{d_{46}} & 0 & 0 & 0 & 0 & \frac{d_{46}-\delta_{6}}{d_{46}}\end{array}\right)$

and

$$
\mathbf{b}=\left(\begin{array}{l}
\delta_{1}\left(d_{12}-\delta_{1}\right) \\
\delta_{2}\left(d_{45}-\delta_{2}\right) \\
\delta_{3}\left(d_{23}-\delta_{3}\right) \\
\delta_{4}\left(d_{56}-\delta_{4}\right) \\
\delta_{5}\left(d_{13}-\delta_{5}\right) \\
\delta_{6}\left(d_{46}-\delta_{6}\right)
\end{array}\right)
$$

If $\operatorname{det}(\mathbf{A}) \neq 0$, there is a one-to-one correspondence between $\left(m_{1}^{2}, \ldots, m_{6}^{2}\right)$ and $\left(l_{1}^{2}, \ldots, l_{6}^{2}\right)$. Remind that $\mathbf{A}$ is 


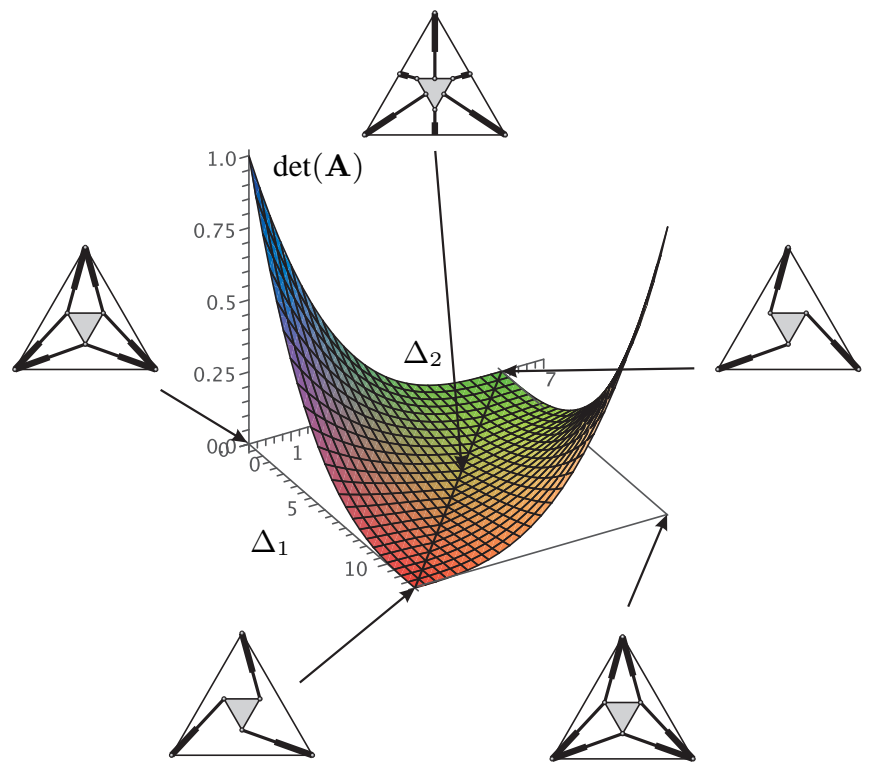

Fig. 7. By properly choosing the offsets $\Delta_{1}=\delta_{1}=\delta_{3}=\delta_{5}$ and $\Delta_{2}=\delta_{2}=\delta_{4}=\delta_{6}$ in Fig 6 , it is possible to reach architecturally singular platforms including the obvious situations in which couples of legs coincide and the architecturally singular Griffis-Duffy platform.

constant as it only depends on architectural parameters. Next, the resulting 6-6 platform is analyzed in more detail through an example.

\section{EXAMPLE}

Let us consider a parallel manipulator with the same topology as the one depicted in Fig. 6 with the following architectural parameters: $d_{12}=d_{23}=d_{13}=12, d_{46}=$ $d_{45}=d_{56}=6, \Delta_{1}=\delta_{1}=\delta_{3}=\delta_{5}$, and $\Delta_{2}=\delta_{2}=$ $\delta_{4}=\delta_{6}$. Substituting these values in (9) and computing its determinant, we obtain

$$
\begin{aligned}
\operatorname{det}(\mathbf{A})= & -\frac{1}{20736} \Delta_{1}^{3} \Delta_{2}^{2}-\frac{1}{10368} \Delta_{1}^{2} \Delta_{2}^{3}+\frac{1}{3456} \Delta_{1}^{3} \Delta_{2} \\
& +\frac{1}{576} \Delta_{1}^{2} \Delta_{2}^{2}+\frac{1}{864} \Delta_{1} \Delta_{2}^{3}-\frac{1}{1728} \Delta_{1}^{3} \\
& -\frac{1}{96} \Delta_{1}^{2} \Delta_{2}-\frac{1}{48} \Delta_{1} \Delta_{2}^{2}-\frac{1}{216} \Delta_{2}^{3}+\frac{1}{48} \Delta_{1}^{2} \\
& +\frac{1}{8} \Delta_{1} \Delta_{2}+\frac{1}{12} \Delta_{2}^{2}-\frac{1}{4} \Delta_{1}-\frac{1}{2} \Delta_{2}+1 .
\end{aligned}
$$

Fig. 7 plots $\operatorname{det}(\mathbf{A})$ as a function of $\Delta_{1}$ and $\Delta_{2}$. When $\Delta_{1}+\Delta_{2}=12$, the introduced offsets lead to an architecturally singular platform because $\operatorname{det}(\mathbf{A})=0$. Now, let us set $\Delta_{1}=\frac{12}{5}$ and $\Delta_{2}=\frac{6}{5}$, and let us suppose that we want to compute the forward kinematic solutions of the resulting robot for the following leg lengths

$$
\begin{aligned}
m_{1} & =\frac{6}{25} \sqrt{6170}, & m_{3} & =\frac{1}{5} \sqrt{7349} \\
m_{5} & =\frac{1}{25} \sqrt{136210}, & m_{2} & =\frac{6}{5} \sqrt{221}, \\
m_{4} & =\frac{1}{50} \sqrt{674605}, & m_{6} & =\frac{1}{5} \sqrt{8153} .
\end{aligned}
$$

Then, substituting these values in (8), it can be verified that this problem is equivalent to solve the forward kinematics of the octahedral manipulator defined by $P_{1}, \ldots, P_{6}$ (see Fig. 6) with leg lengths

$$
\begin{array}{lll}
l_{1}=\frac{198}{10}, & l_{2}=18, & l_{3}=18, \\
l_{4}=17, & l_{5}=\frac{149}{10}, & l_{6}=\frac{178}{10},
\end{array}
$$

which is the same problem as the one analyzed in [12]. Substituting the above values in equation (3) we get

$$
\begin{aligned}
& \frac{1}{100\left(s_{1,5}-25\right)\left(s_{1,5}-841\right)\left(25 s_{1,5}-4761\right)\left(25 s_{2,3}-16641\right)} \\
& \left(375625 s_{1,5}^{4}+2425914325 s_{1,5}^{3}-2781440777549 s_{1,5}^{2}\right. \\
& +929177720979831 s_{1,5}-94994611164672840 \\
& \pm 24\left(25 s_{1,5}-4761\right)\left(25 s_{1,5}-16641\right) \sqrt{s_{1,5}^{2}-757 s_{1,5}+27325} \\
& \sqrt{2220100 s_{1,5}^{2}-1689044183 s_{1,5}+277445877004} \\
& \pm 21600\left(s_{1,5}-25\right)\left(s_{1,5}-841\right) \sqrt{625 s_{1,5}^{2}-495325 s_{1,5}+85928301} \\
& \left.\sqrt{625 s_{1,5}^{2}-465550 s_{1,5}+66028321}\right)=0 .
\end{aligned}
$$

Twice squaring the above equation, the following 8th order polynomial is obtained

$$
\begin{aligned}
& 2.5720 s_{1,5}^{8}-7719.2542 s_{1,5}^{7}+1.0078 \cdot 10^{7} s_{1,5}^{6} \\
& -7.4833 \cdot 10^{9} s_{1,5}^{5}+3.4607 \cdot 10^{12} s_{1,5}^{4} \\
& -1.0220 \cdot 10^{15} s_{1,5}^{3}+1.8843 \cdot 10^{17} s_{1,5}^{2} \\
& -1.9854 \cdot 10^{19} s_{1,5}+9.1598 \cdot 10^{20}=0 .
\end{aligned}
$$

This polynomial has six real roots: $269.2,328.7,359.5$, 463.6, 497.9, and 513.0. Each of them leads to two mirror poses with respect to the base plane. The resulting poses for the case in which $P_{1}=(0,0,0)^{T}, P_{2}=(6, \sqrt{108}, 0)^{T}$, and $P_{3}=(12,0,0)^{T}$, appear in Fig. 8 where the mirror reflections with respect to the base plane are not represented.

\section{CONCLUSION}

Stating the kinematics analysis of the octahedral manipulator in terms of poses introduces two major disadvantages: (a) a reference frame has to be introduced, and (b) all formulas involve translations and rotations simultaneously. This paper proposes a different approach in which, instead of directly computing the sought Cartesian poses, a problem fully posed in terms of distances is first solved. Then, the original problem can be trivially solved by sequences of trilaterations.

The presented distance-based formulation also permits to generate a family of Stewart-Gough platforms whose members are kinematically equivalent to the octahedral manipulator. One of this members has no double-ball-joints and, hence, its important technological interest. Future developments in which an octahedral manipulator is required but double-ball-joints have to be avoided can benefit from this result. 


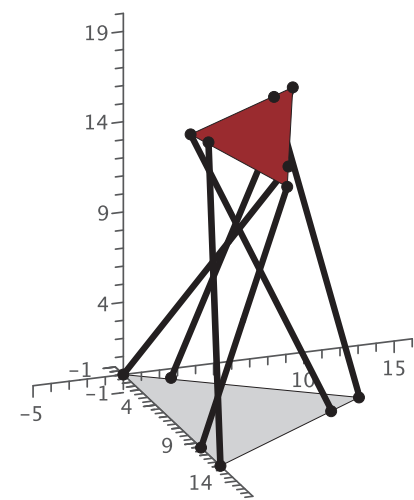

$s_{1,5}=269.2$

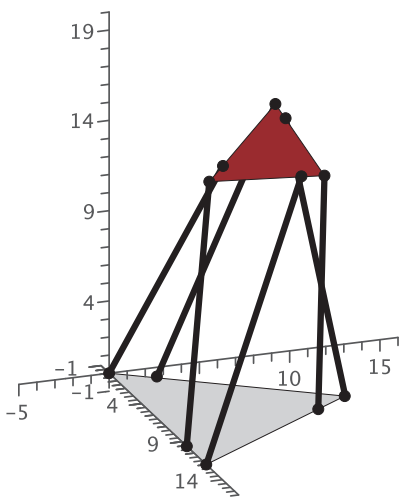

$s_{1,5}=463.5$

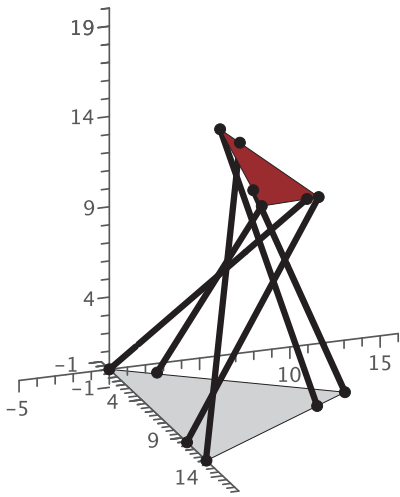

$s_{1,5}=328.7$

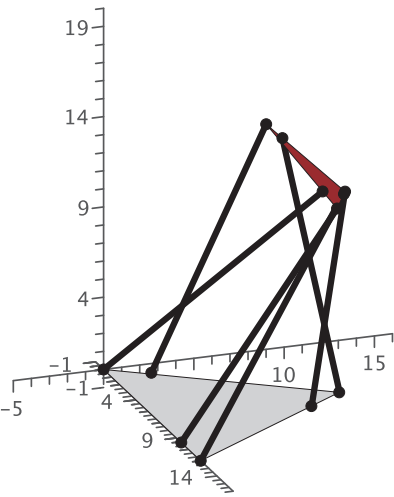

$s_{1,5}=497.9$

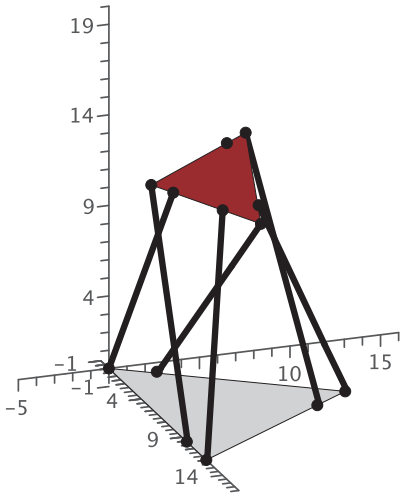

$s_{1,5}=359.5$

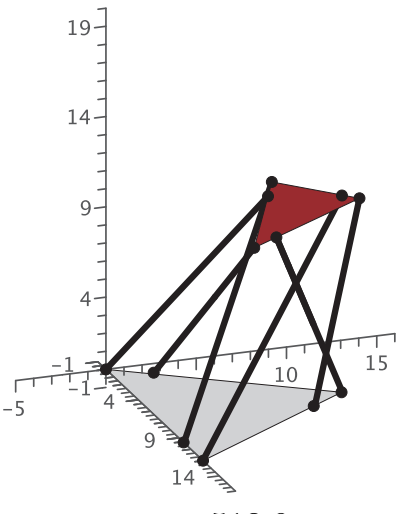

$s_{1,5}=513.0$

Fig. 8. The forward kinematic solutions of the analyzed example. The mirror reflections with respect to the base plane are not included.

\section{REFERENCES}

[1] J.M. Porta, L. Ros, and F. Thomas, "Inverse kinematics by distance matrix completion," International Workshop on Computational Kinematics, May 4-6, Cassino, Italy, 2005.

[2] B. Dasgupta and T. S. Mruthyunjaya, "The Stewart platform manipulator: a review," Mechanism and Machine Theory, Vol. 35, No. 1, pp. $15-40,2000$.

[3] K.H. Hunt and P.R. McAree, "The octahedral manipulator: geometry and mobility," The International Journal of Robotics Research, Vol. 17, No. 8, pp. 868-885, 1998

[4] P. Bosscher and I. Ebert-Uphoff, "A novel mechanisms for implementing multiple colocated spherical joints," Proc. of the IEEE International Conference on Robotics and Automation, pp. 336-341, 2003.

[5] J. Lee, J. Duffy, and K.H. Hunt, "A practical quality index based on the octahedral manipulator," The International Journal of Robotics Research, Vol. 17, No. 10, pp. 1081-1090, 1998.

[6] K. J. Waldron, M. Raghavan and B. Roth, "Kinematics of a hybrid series-parallel manipulation system," ASME Journal of Dynamic Systems, Measurement, and Control, Vol. 111, No. 2, pp. 211-221, 1989.

[7] G.J. Hamlin and A.C. Sanderson, Tetrobot: A Modular Approach to Reconfigurable Parallel Robotics, Kluwer Academic Publishers, Norwell, MA, 1998.

[8] R.S. Stoughton and T. Arai, "A modified Stewart platform manipulator with improved dexterity," IEEE Trans. on Robotics and Automation, Vol. 9, No. 2, pp. 166-173, 1993.

[9] M. Griffis and J. Duffy, "Method and apparatus for controlling geometrically simple parallel mechanisms with distinctive connections," US Patent 5, 179,525, 1993.

[10] F. Thomas and L. Ros, "Revisiting trilateration for robot localization," IEEE Transactions on Robotics, Vol. 21, No. 1, pp. 93-101, 2005.

[11] P. Nanua, K. Waldron, and V. Murthy, "Direct kinematic solution of a Stewart platform," IEEE Transactions on Robotics and Automation, Vol. 6, No. 4, pp. 438-444, 1990.

[12] M. Griffis and J. Duffy, "A forward displacement analysis of a class of Stewart platforms," Journal of Robotic Systems, Vol. 6, pp. 703-720, 1989.

[13] C. Innocenti and V. Parenti-Castelli, "Direct position analysis of the Stewart platform mechanism", Mechanism and Machine Theory, Vol. 25, No. 6, pp. 611 - 621, 1990.

[14] I.D. Akçali and M.Mutlu, "A novel approach in the direct kinematics of Stewart platform mechanisms with planar platforms," Thans. of the ASME, Journal of Mechanical Dessign, Vol. 128, No. 1, pp. 252-263, 2006.

[15] K. Liu, J.M. Fitzgerald, F.L. and Lewis, "Kinematic analysis of a Stewart platform manipulator," IEEE Trans. on Industrial Electronics, Vol. 40, No. 2, pp. 282-293, 1993.

[16] D-M. Ku, "Direct displacement analysis of a Stewart platform mechanism", Mechanism and Machine Theory, Vol. 34, No. 3, pp. 453-465, 1999.

[17] S-K. Song and D-S. Kwon, "Efficient formulation approach for the forward kinematics of the Stewart-Gough plaform," Proc. of the 2001 IEEE/RSJ International Conference on Inteligent Robots and Systems, pp. 1688-1693.

[18] J-P. Dedieu and G. Norton, "Stewart varieties: a direct algebraic model for Stewart platforms," SIGSAM Bulletin, Vol. 24, No. 4, pp. 42-59, 1990.

[19] D.M. Downing, A.E. Samuel, and K.H. Hunt, "Identification of the special configurations of the octahedral manipulator using the pure condition," The International Journal of Robotics Research, Vol. 21, No. 2, pp. 147-159, 2002.

[20] J. Borràs, F. Thomas, and C. Torras, "On $\Delta$-transforms," IEEE Transactions on Robotics, Vol. 25, No. 6, pp. 1225-1236, 2009.

[21] J. Borràs and F. Thomas, "On the primal and dual forms of the Stewart platform pure condition," submitted for publicaction. 